\title{
Intersystem crossing dynamics in thionated uracils studied by time-resolved photoelectron spectroscopy: The effect of substituent position
}

\author{
Susanne Ullrich, Abed Mohamadzade \\ Department of Physics and Astronomy, University of Georgia, Athens GA 30602, USA
}

\begin{abstract}
The photophysics of thionated uracils are investigated using time-resolved photoelectron spectroscopy with emphasis on evaluating differences in intersystem crossing dynamics with respect to substituent position.
\end{abstract}

\begin{abstract}
1. Introduction
The canonical nucleobases are known to undergo ultrafast excited state relaxation to the ground state, either directly or via intermediate singlet excited states. Structurally modified nucleobases, such as thiobases, where one oxygen atom is replaced by sulphur, provide an excellent example how drastically single-atom substitution can affect the deactivation mechanism. Recent time-resolved photoelectron spectroscopy (TRPES) studies of 2-thiouracil (2-TU) observed surprisingly fast and efficient intersystem crossing from the $S_{1}\left(n \pi^{*}\right)$ to the triplet manifold and subsequently back to the ground state $[1,2]$. This contrasts the photophysics of uracil which predominantly follow above-mentioned, singlet-only relaxation pathways with only a minor fraction reaching and becoming trapped in a long-lived triplet state [1]. In direct extension of this work, the TRPES spectrum of 4-thiouracil (4-TU) is presented here to investigate the effect of substituent position on the photodynamics of thionated uracils. Strikingly different intersystem crossing dynamics compared to 2-TU are observed. It is noteworthy, that the triplet state dynamics of thiobases are of interest to phototherapeutic applications that rely on long-lived, reactive triplet states for the generation of singlet oxygen with cytotoxic action.
\end{abstract}

\section{Experimental Details}

The apparatus consists of an amplified femtosecond laser system and a magnetic bottle photoelectron spectrometer which have been described in detail elsewhere [1,2]. 2-TU and 4-TU are heated inside a quartz holder and transferred into a continuous molecular beam. The UV pump and time-delayed probe pulses intersect with the molecular beam in the ionization region of the spectrometer where the molecules undergo a 1+2' excitation-ionization process. Photoelectron kinetic energies are recorded at different pump-probe delays to acquire a time-resolved photoelectron spectrum. Probe wavelengths below the onset of the UV absorption spectra (330nm for 2-TU; 400nm for 4-TU) and two-photon ionization are chosen to avoid contributions from unwanted probe-pump signals within the crosscorrelation region.

\section{Results and Discussion}

Fig. 1 shows the TRPES spectra of 2-TU (top) and 4-TU (bottom) following photoexcitation close to their absorption maximum at 283 and 330nm, respectively. The TRPES spectra were recorded over extended pump-probe delays to capture the slower intersystem crossing dynamics, but $2 \mathrm{~d}$ plots in Fig. 1 are limited to the first $\sim 4$ ps where distinct spectral changes are observed. The white lines, $\mathrm{n}^{-1}$ and $\pi^{-1}$, indicate the two lowest, vertical IPs from $\mathrm{S}_{0}$, corresponding to $\mathrm{n}$ - and $\pi$-holes that are mostly localized on the sulphur atom [3]. For the decay dynamics, a fitting function consisting of three sequential exponential decays, convoluted with the Gaussian instrument response function, is needed to accurately describe the experimental data. The timetraces and their three fitted contributions are plotted in Fig. 1 (right) for the entire pump-probe range.

Initial photoexcitation of 2 -TU and 4-TU proceeds to a vibrationally excited $\mathrm{S}_{2}\left(\pi \pi^{*}\right)$ state which preferentially ionizes into a $\pi$-hole. Hence, around zero pump-probe delay a photoelectron band with ionization into $\operatorname{IP}\left(\pi^{-1}\right)$ is observed. The photoelectron band decays within $<75 \mathrm{fs}$ and is associated with the lifetime of the $S_{2}\left(\pi \pi^{*}\right)$ state. During internal conversion to the $S_{1}\left(n \pi^{*}\right)$ state, which preferentially ionizes into $\operatorname{IP}\left(\mathrm{n}^{-1}\right)$, the molecule gains additional vibrational energy of up to $0.5-1 \mathrm{eV}[1,2]$. Upon ionization, vibrational excitation is transferred to the cationic state which significantly shifts the photoelectron band towards higher electron binding energies compared to the vertical IP $\left(n^{-1}\right)$. The $S_{1}\left(n \pi^{*}\right)$ state subsequently facilitates intersystem crossing to the close-by triplet manifold, but, according to our measurements, proceeds much slower in 4-TU. This observation confirms ab initio calculations that predict larger spin orbit coupling constants for 2-TU [4]. Once on the triplet manifold 2-TU and 4-TU show strikingly different

Corresponding author: ullrich@physast.uga.edu 
behaviour. Intersystem crossing back to the ground state occurs surprisingly fast in 2-TU and is supported by ab initio calculations that predict a very small barrier to the $\mathrm{T}_{1} / \mathrm{S}_{0}$ crossing point and a large spin orbit coupling constant [1]. 4-TU, in contrast, gets trapped in a long-lived triplet state indicating that intersystem crossing back to the ground state is energetically not favoured [4].
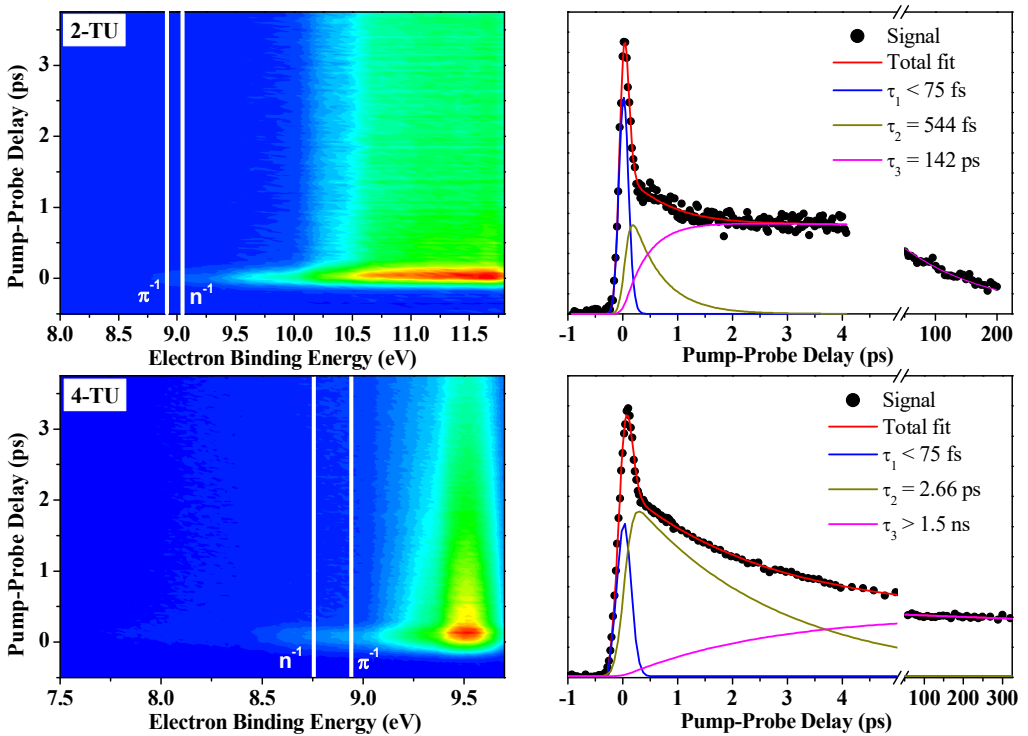

Fig. 1. TRPES spectra of 2-TU (top, left) and 4-TU (bottom, left) following photoexcitation close to their UV absorption maximum. The $2 \mathrm{~d}$ plots are truncated to a pump-probe range of $\sim 4 \mathrm{ps}$ for clarity; photoelectron spectra are plotted as a function of electron binding energy, which is calculated as the total photon energy minus the measured photoelectron kinetic energy, for direct comparison to ionization potentials (indicated as white lines). 2 TU: $\operatorname{IP}\left(\pi^{-1}\right)=8.891 \mathrm{eV}, \operatorname{IP}\left(\mathrm{n}^{-1}\right)=9.013 \mathrm{eV} ; 4-\mathrm{TU}: \operatorname{IP}\left(\mathrm{n}^{-1}\right)=8.75 \mathrm{eV}, \operatorname{IP}\left(\pi^{-1}\right)=8.918 \mathrm{eV}[3]$. The associated timetraces (right) were extracted assuming sequential decay dynamics of three exponentials convoluted with the Gaussian instrument response function.

\section{Conclusions}

This TRPES study presents the first measurements of the photophysics of thionated uracils in the gas phase. While the relaxation dynamics of 2-TU and 4-TU follow a generally similar pathway involving $\mathrm{S}_{2}\left(\pi \pi^{*}\right) \rightarrow \mathrm{S}_{1}\left(\mathrm{n} \pi^{*}\right) \rightarrow$ triplet manifold $\rightarrow \mathrm{S}_{0}$, the position of the sulphur atom profoundly alters their intersystem crossing dynamics with significantly longer timeconstants in the case of 4-TU. Intersystem crossing in 2-TU is among the fastest reported in the literature to date [2], whereas 4-TU shows trapping in a long-lived (ns or longer) triplet state.

\section{Acknowledgement}

This work was supported by the National Science Foundation grant CHE-1362237.

\section{References}

[1] H. Yu, J. A. Sánchez-Rodríguez, M. Pollum, C. E. Crespo-Hernández, S. Mai, P. Marquetand, L. González, and S. Ullrich, "Internal conversion and intersystem crossing pathways in UV excited, isolated uracils and their implications in prebiotic chemistry," Phys. Chem. Chem. Phys. 18, 20168 (2016).

[2] J. A. Sánchez-Rodríguez, A. Mohamadzade, S. Mai, B. Ashwood, M. Pollum, P. Marquetand, L. González, C. E. Crespo-Hernández, and S. Ullrich, "2-Thiouracil intersystem crossing photodynamics studied by wavelength-dependent photoelectron and transient absorption spectroscopies," Phys. Chem. Chem. Phys. 19, 19756 (2017)

[3] M. Ruckenbauer, S. Mai, P. Marquetand, and L. González, "Photoelectron spectra of 2-thiouracil, 4-thiouracil, and 2,4-dithiouracil," J. Chem. Phys. 144, 074303 (2016).

[4] S. Arslancan, L. Martínez-Fernández, and I. Corral, "Photophysics and Photochemistry of Canonical Nucleobases' Thioanalogs: From Quantum Mechanical Studies to Time Resolved Experiments," Molecules 22, 998 (2017). 